\title{
Contribuição da aplicação do ultrassom de baixa potência na prevenção de osteopenia em tíbias de ratos sob ausência de carga
}

\author{
[The contribution of the application of low power ultrasound to the prevention of rat tibial osteopenia \\ under the absence of load] \\ C.H.M. Borges ${ }^{1}$, J.C. Apolinário ${ }^{1}$, P.L. Florindo ${ }^{2}$, M.J.Q. Louzada ${ }^{2} *$ \\ ${ }^{1}$ Aluna de pós-graduação - Universidade Estadual Paulista - Araçatuba, SP \\ ${ }^{2}$ Universidade Estadual Paulista - Araçatuba, SP
}

\begin{abstract}
RESUMO
Este trabalho objetivou verificar se o ultrassom de baixa potência (US) previne a ocorrência de osteopenia em tíbias de ratos sob ausência de carga. Foram utilizados 45 Rattus novergicus albinus, Wistar adultos, machos, distribuídos em cinco grupos iguais: C - animais-controle livres em gaiolas por 21 dias; $\mathrm{S}$ animais suspensos pela cauda por 21 dias; ST - suspensos pela cauda por 21 dias e concomitantemente tratados com US; $\mathrm{S} \rightarrow \mathrm{C}$ - suspensos por 21 dias e depois permanecendo livres em gaiolas por mais 21 dias; $\mathrm{S} \rightarrow \mathrm{CT}$ - suspensos por 21 dias e depois permanecendo livres em gaiolas por mais 21 dias e concomitantemente tratados com US. O tratamento foi realizado com US de $1,5 \mathrm{MHz}$, ciclo de trabalho $1: 4,30 \mathrm{~mW} / \mathrm{cm}^{2}$, na tíbia direita, por 15 sessões de 20 minutos cada, cinco sessões por semana. Ainda vivos, os animais foram submetidos a exame de densitometria óssea para verificação da densidade mineral óssea (DMO) e do conteúdo mineral ósseo (CMO). Após a eutanásia dos animais, as tíbias foram desarticuladas, dissecadas e submetidas a ensaio mecânico destrutivo para análise da força máxima (Fmáx) e da rigidez (R). Foram avaliados também o comprimento (L) e o diâmetro (D) no ponto médio da tíbia. O grupo $\mathrm{S}$ apresentou valores de DMO, CMO, Fmáx, R, L e D menores em relação ao grupo C, demonstrando que a suspensão pela cauda é prejudicial a estas variáveis. O tratamento dos animais suspensos com o US, grupo ST, elevou os valores de CMO e DMO em relação aos do grupo S, igualando-os aos do grupo C. A Fmáx, R e L do grupo ST aumentou em relação ao grupo $\mathrm{S}$ e também em relação ao grupo $C(p<0,05)$. Nenhuma diferença significativa foi encontrada entre as variáveis analisadas para os grupos $\mathrm{S} \rightarrow \mathrm{CT}$ e $\mathrm{S} \rightarrow \mathrm{C}(\mathrm{p}>0,05)$. Os resultados obtidos neste estudo permitem concluir que o US de baixa potência contribuiu na prevenção e reversão da ocorrência da osteopenia nos animais submetidos à suspensão pela cauda, demonstrando que a ausência do estímulo mecânico causada pela impossibilidade da deambulação pode ser minimizada pela ação mecânica deste.
\end{abstract}

Palavras-chave: estimulação física, osteopenia, osteoporose, prevenção, ultrassom

\begin{abstract}
This work intended to verify if the low power ultrasound (US) prevents the occurrence of osteopenia on the tibia of rats under the absence of load. 45 adult male Wistar Rattus novergicus albinus were used, separated in five equal groups: $C$ - control free animals in cages for 21 days; $S$-animals suspended by the tail for 21 days; ST - animals suspended by the tail for 21 days and concurrently treated with US; $S \rightarrow C$ - animals suspended for 21 days and then free in cages for another 21 days; $S \rightarrow C T$ - animals suspended for 21 days and then free in cages for another 21 days and concurrently treated with US. The treatment was performed with US with $1.5 \mathrm{MHz}$, work cycle $1: 4,30 \mathrm{~mW} / \mathrm{cm}^{2}$, on tibia, for 15 sessions of 20 minutes each, 5 sessions per week. While still alive, the animals underwent bone densitometry examination to verify the Bone Mineral Density (BMD) and the Bone Mineral Content (BMC). After the euthanasia of the animals, the tibias were disjointed, dissected and underwent destructive mechanical
\end{abstract}

Recebido em 1 de agosto de 2012

Aceito em 16 de outubro de 2013

*Autor para correspondência (corresponding author)

E-mail: louzada@fmva.unesp.br 
testing for the analysis of the maximum force (Fmax) and Rigidity $(R)$. The length $(L)$ and the diameter (D) of the middle point of the tibia were also measured. In group $S, B M D, B M C, F m a x, R, L$ and $D$ were smaller than in group $C$, demonstrating that the suspension by the tail is detrimental to these variables. The treatment of the suspended animals with the US, in group ST, increased the BMD and BMC compared to group $S$, leveling them to group $C$. The Fmax, $R$ and $L$ of group ST increased compared to group $S$ and compared to group $C$ as well $(p<0.05)$. No significant difference was found between the measured variables for the groups $S \rightarrow C T$ and $S \rightarrow C$ ( $p>0.05)$. The results obtained in this study lead to the conclusion that the low power US contributes to the prevention of the occurrence of osteopenia on animals under suspension by the tail, showing that the absence of the mechanical stimulation caused by the impossibility of deambulation can be minimized by its action.

Keywords: physical stimulation, osteopenia, osteoporosis, prevention, ultrasound

\section{INTRODUÇÃO}

A osteoporose é uma doença que acomete a população humana há milhares de anos (Dequerker et al., 1997), sendo considerado um problema de saúde pública (Engermann et al., 2005). Tornou-se uma das doenças osteometabólicas mais comuns e responsável por alto índice de morbidade e mortalidade entre os idosos, com enormes repercussões sociais e econômicas, provocando grande impacto na qualidade de vida e no grau de dependência dos indivíduos acometidos (Forsbach et al., 1994). Além disso, é um problema clínico e social, pois pode dificultar o desenvolvimento das atividades cotidianas, influenciando o bem-estar e a qualidade de vida relacionada à saúde (QVRS) (Aranha et al., 2006). Calcula-se que um terço das mulheres brancas no mundo com idade superior a 65 anos estão acometidas por essa enfermidade, sendo definida como a "epidemia do século 21" (Navega et al., 2007). A osteopenia é o estágio inicial ou antecessor da osteoporose e caracteriza-se pela diminuição da massa óssea, causada pela perda de cálcio (Vargas et al., 2003).

Ao longo da vida, o tecido ósseo é continuamente renovado em um processo extremamente regulado, chamado de remodelamento ósseo (Nordin et al., 2003). Por ser um tecido altamente vascularizado, o osso tem capacidade de alterar suas propriedades e geometria em resposta às mudanças na demanda mecânica (Nordin et al., 2003; Lirani et al., 2004).

A remodelação óssea e sua estimulação são motivos de grande número de estudos, com interesse focado principalmente nos fatores capazes de acelerar a osteointegração, promovendo maior rapidez na reabilitação do paciente. Estudos demonstram que a resposta de reparo ósseo pode ser acentuada pela estimulação física, eletromagnética e mecânica (Lind e Bunger, 2001). O ultrassom de baixa intensidade é uma estimulação mecânica e vem sendo utilizado há algum tempo para acelerar o processo de regeneração óssea, aproveitando-se do efeito piezoelétrico, propriedade característica do tecido ósseo (Azuma et al., 2001; Apolinário et al., 2008).

O mecanismo de piezoeletricidade é um advento físico característico de certos materiais que, quando deformados por uma ação mecânica, desenvolvem cargas elétricas em sua superfície. O contrário também é verdadeiro, ou seja, ao se colocar um material piezoelétrico sob um campo elétrico, este material sofrerá deformação mecânica (Okuno et al., 1986).

Um dos fatores responsáveis pela manutenção do metabolismo mineral normal dos ossos nos membros pélvicos e torácicos dos animais é a compressão longitudinal exercida sobre eles, produzida pelo próprio peso corporal (Johnson, 1998). Esta pressão estimula o crescimento ósseo por aposição. A ação do peso corporal resulta em maior espessura do osso e maior densidade mineral da diáfise (McArdle et al., 2011). A exposição a ambientes de microgravidade afeta diretamente o sistema musculoesquelético, alterando seu metabolismo. Esse ambiente gera uma baixa tensão sobre os ossos, e, por consequência, menor massa óssea será necessária para manter a integridade estrutural do esqueleto (Johnson, 1998).

Alguns pesquisadores afirmaram que o modelo ideal para simular atrofia muscular e o desgaste ósseo seria aquele que permitisse livre 
mobilidade ao animal, porém com restrição aos movimentos dos membros pélvicos (Musacchia et al., 1983). O modelo utilizado por Apolinário et al. (2008) foi o de suspender os animais pela cauda. Nessa situação, os membros pélvicos dos animais permanecem com livre movimentação, porém sem contato com o solo.

Um dos fatores fundamentais para estabelecer o quadro de desmineralização óssea é a determinação da densidade mineral óssea (DMO), que fornece parâmetros que podem definir o risco de fraturas patológicas. A densitometria óssea é a análise utilizada para determinar a DMO e diagnosticar a osteopenia e a osteoporose (Mello et al., 2012). O densitômetro de dupla emissão de raios-X (DXA) é a técnica considerada "padrão ouro" para este tipo de análise, pois, em razão de sua precisão, tempo de realização e segurança, fornece dados precisos neste tipo de diagnóstico. O exame de densitometria óssea estabelece valores do conteúdo mineral do osso - CMO específico - num momento único no tempo. Este valor pode determinar se há osteoporose e se a densidade é baixa o suficiente para tornar o osso susceptível à fratura.

Devido ao constante interesse em desenvolver meios e técnicas que auxiliem na prevenção de doenças osteometabólicas, foi objetivo deste estudo avaliar, pelas análises densitométrica e biomecânica, se o ultrassom de baixa intensidade previne a ocorrência da osteopenia em tíbias de ratos submetidos à ausência de carga.

\section{MATERIAL E MÉTODOS}

Após a aprovação pelo Comitê de Ética em Pesquisa da Faculdade de Odontologia da Unesp - Campus de Araçatuba (Protocolo n ${ }^{\circ}$ 633/10), 45 ratos machos, Rattus novergicus albinus, linhagem Wistar, adultos, com três meses de idade e massa corporal média de $367 \pm 36 \mathrm{~g}$, foram alojados em gabinetes de criação com temperatura ambiente controlada $\left(22 \pm 2^{\circ} \mathrm{C}\right)$, umidade de $65 \%$, ciclo claro/escuro (12/12 horas diárias) e filtragem de ar. Os animais permaneceram em gaiolas comuns com número máximo de cinco em cada uma, sendo alimentados com ração Purina-Labina ${ }^{\circledR}$ e água potável à vontade.
Aos três meses de idade, os animais foram distribuídos aleatoriamente em cinco grupos: $\mathrm{C}-$ grupo de animais-controle que permaneceram livres em gaiolas por 21 dias e então foram eutanasiados; S - grupo de animais suspensos pela cauda por 21 dias e em seguida eutanasiados; ST - grupo de animais suspensos pela cauda por 21 dias com simultâneo tratamento com US e em seguida eutanasiados; $\mathrm{S} \rightarrow \mathrm{C}$ - grupo de animais suspensos por 21 dias e que, em seguida, permaneceram livres na caixa por mais 21 dias e então foram eutanasiados; $\mathrm{S} \rightarrow \mathrm{CT}$ - grupo de animais suspensos por 21 dias e que, em seguida, permaneceram livres na caixa por mais 21 dias com simultâneo tratamento com US e então foram eutanasiados.

Para a realização da suspensão, os animais foram anestesiados com ketamina $(30 \mathrm{mg} / \mathrm{kg})$ e xilazina $(3 \mathrm{mg} / \mathrm{kg})$, via intramuscular. Adotou-se a mesma técnica utilizada por Apolinário et al., 2008, a qual, segundo Morey-Holton e Globus (1998), provoca um mínimo estresse ao animal. Com os animais anestesiados, a cauda foi lavada com sabonete antisséptico (com pH neutro) e, depois de seca, envolvida com espuma adesiva Reston ${ }^{\circledR}$ desde a base caudal até aproximadamente a $20^{\mathrm{a}}$ vértebra. Em seguida, a cauda era envolvida uniformemente com faixa elástica Coban, permitindo circulação sanguínea necessária a fím de evitar necrose e proporcionar o mínimo desconforto possível ao animal. Após este processo, uma fita adesiva esparadrapo foi colocada para fixar o cadarço sarjado de aproximadamente $7 \mathrm{~cm}$, para formar uma alça e, assim, conectar o animal ao sistema de suspensão. Por fim, foi realizada a caudectomia entre a $17^{\mathrm{a}}$ e a $20^{\mathrm{a}}$ vértebra caudal, de modo a ter o mínimo de atrito com a presilha, evitando escoriações na cauda. Após a suspensão, foi feito curativo com iodo povedine por 72 horas.

Durante o tratamento, os animais foram imobilizados manualmente para que a tíbia do antímero direito fosse tratada. O tratamento foi realizado com US pulsado de baixa intensidade, frequência de $1,5 \mathrm{MHz}$, ciclo de trabalho $1: 4$, $30 \mathrm{~mW} / \mathrm{cm}^{2}$ (Apolinário et al., 2011). No total, foram feitas 15 sessões de US, de 20 minutos cada por dia (Gonçalves et al., 2007), sendo cinco dias consecutivos de tratamento com dois dias de intervalo para o próximo tratamento, sempre no mesmo horário. O protocolo de tratamento teve início para o ST 24 horas após 
suspensão dos animais e para o $\mathrm{S} \rightarrow \mathrm{CT} 24$ horas após os animais serem colocados livres na caixa.

Após o período experimental, os animais foram eutanasiados com dose excessiva de ketamina e xilazina, e suas tíbias foram retiradas para posterior análise densitométrica e biomecânica.

Com o animal vivo, anestesiado com ketamina $(30 \mathrm{mg} / \mathrm{kg}) \quad$ e xilazina $(3 \mathrm{mg} / \mathrm{kg})$, via intramuscular, o membro pélvico era posicionado de forma que a análise da DMO fosse realizada em toda a tíbia, utilizando-se densitômetro de dupla emissão de raios-X (DXA) (modelo DPXAlpha, Lunar ${ }^{\circledR}$ ) com software para pequenos animais.

Após a eutanásia dos animais, as tíbias foram retiradas, limpas, dissecadas, imersas em soro fisiológico e congeladas a ${ }^{-} 20^{\circ} \mathrm{C}$. Para a realização do ensaio mecânico, as tíbias eram retiradas do freezer e mantidas em ambiente com temperatura de aproximadamente $22^{\circ} \mathrm{C}$ até atingirem o equilíbrio térmico.
As tíbias foram submetidas a ensaio mecânico de flexão a três pontos, em máquina universal EMIC $^{\circledR}$, modelo DL 3000, com célula de carga de 2000N. Permaneciam apoiadas em dois suportes (dois pontos), separados entre si por $3 / 4$ do comprimento original do osso, e a força era aplicada no meio geométrico, entre os dois apoios, na diáfise tibial. A carga foi aplicada a uma velocidade de $5 \mathrm{~mm} / \mathrm{min}$. Foi registrada a força máxima - Fmáx - admitida pelo osso e, em seguida, calculada a rigidez $-\mathrm{R}$ - do osso, obtida pela inclinação da curva Força $(\mathrm{N})$ x Deformação $(\mathrm{mm})$ relativa à fase elástica.

O comprimento total e o diâmetro na região diafisária das tíbias foram medidos com paquímetro de precisão $(0,05 \mathrm{~mm})$.

\section{RESULTADOS}

Os resultados das variáveis analisadas nas tíbias dos grupos experimentais estão apresentados como média \pm desvio-padrão na Tab. 1 e nas Fig. 1 e 2.

Tabela 1. Parâmetros biofísicos (média \pm desvio-padrão) para tíbia de ratos Wistar de animais controle C - livres em gaiolas por 21 dias; S - suspensos pela cauda por 21 dias; ST - suspensos pela cauda por 21 dias e simultaneamente tratados com US; $\mathrm{S} \rightarrow \mathrm{C}$ - suspensos por 21 dias, seguidos de 21 dias livres na caixa; $\mathrm{S} \rightarrow \mathrm{CT}$ - suspensos por 21 dias, seguidos de 21 dias livres na caixa com simultâneo tratamento com US.

\begin{tabular}{lccccc}
\hline & \multicolumn{5}{c}{ Grupos } \\
\cline { 2 - 6 } Parâmetros & $\mathrm{C}$ & $\mathrm{S}$ & $\mathrm{ST}$ & $\mathrm{S} \rightarrow \mathrm{C}$ & $\mathrm{S} \rightarrow \mathrm{CT}$ \\
\cline { 2 - 6 } Área $\left(\mathrm{cm}^{2}\right)$ & $1,727 \pm 0,052 \mathrm{a}, \mathrm{b}$ & $1,657 \pm 0,107 \mathrm{a}, \mathrm{b}$ & $1,822 \pm 0,140 \mathrm{~b}$ & $1,760 \pm 0,129 \mathrm{~b}$ & $1,571 \pm 0,179 \mathrm{a}$ \\
CMO $(\mathrm{g})$ & $0,231 \pm 0,029 \mathrm{a}$ & $0,195 \pm 0,024 \mathrm{~b}$ & $0,232 \pm 0,027 \mathrm{a}$ & $0,229 \pm 0,023 \mathrm{a}, \mathrm{b}$ & $0,250 \pm 0,022 \mathrm{a}$ \\
DMO $\left(\mathrm{g} / \mathrm{cm}^{2}\right)$ & $0,138 \pm 0,019 \mathrm{a}$ & $0,114 \pm 0,010 \mathrm{~b}$ & $0,134 \pm 0,013 \mathrm{a}$ & $0,130 \pm 0,008 \mathrm{a}, \mathrm{b}$ & $0,142 \pm 0,013 \mathrm{a}$ \\
Fmáx $(\mathrm{N})$ & $71,70 \pm 3,11 \mathrm{a}$ & $62,18 \pm 4,74 \mathrm{~b}$ & $83,16 \pm 7,55 \mathrm{c}$ & $81,12 \pm 8,53 \mathrm{a}, \mathrm{c}$ & $78,94 \pm 9,03 \mathrm{a}, \mathrm{c}$ \\
$\mathrm{R}\left(\mathrm{x} 10^{-3} \mathrm{~N} / \mathrm{m}\right)$ & $122,19 \pm 10,12 \mathrm{a}$ & $96,12 \pm 8,27 \mathrm{~b}$ & $156,46 \pm 29,30 \mathrm{c}$ & $153,99 \pm 22,60 \mathrm{c}$ & $137,36 \pm 20,08 \mathrm{a}, \mathrm{c}$ \\
\hline
\end{tabular}

CMO - Conteúdo mineral ósseo; DMO - densidade mineral óssea; Fmáx - força máxima admitida pelo osso; $\mathrm{R}$ - rigidez óssea. *a,b,c,d - Médias seguidas de letras iguais, na mesma linha, implicam ausência de diferença estatística ( $p>0,05)$ entre os grupos.

Na Fig. 1, estão apresentados os valores relativos ao diâmetro das tíbias dos ratos. Houve diferença $(\mathrm{p}<0,05)$ entre os grupos $\mathrm{C}$ e $\mathrm{S}, \mathrm{S} \rightarrow \mathrm{C}$ e $\mathrm{S} \rightarrow \mathrm{CT}$, e não houve diferença estatística $(p>0,05)$ entre os grupos $\mathrm{S}$ e $\mathrm{ST}, \mathrm{S} \rightarrow \mathrm{C}$ e $\mathrm{S} \rightarrow \mathrm{CT}$. Isto demonstra que a suspensão influenciou negativamente no diâmetro das tíbias.
Na Fig. 2, estão os valores do comprimento das tíbias dos ratos. Houve diferença estatística entre os grupos $\mathrm{C}, \mathrm{S}$ e $\mathrm{ST}$, também entre $\mathrm{S}, \mathrm{ST}$ e $\mathrm{S} \rightarrow \mathrm{C}$, e entre $\mathrm{ST}, \mathrm{S} \rightarrow \mathrm{C}$ e $\mathrm{S} \rightarrow \mathrm{CT}$. Não houve diferença estatística entre os grupos $\mathrm{S} \rightarrow \mathrm{C}$ e $\mathrm{S} \rightarrow \mathrm{CT}$ 


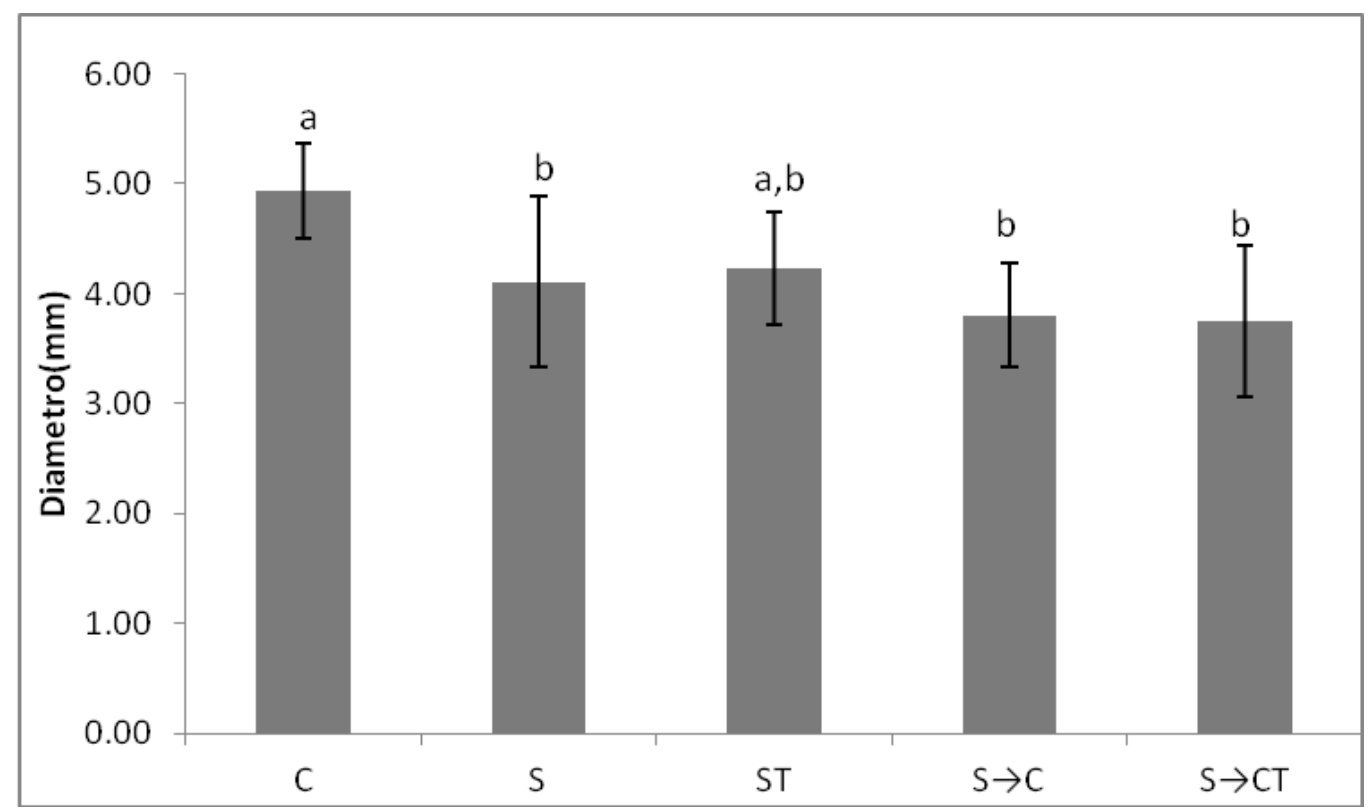

Figura 1. Diâmetro das tíbias (média \pm desvio-padrão) de ratos Wistar de animais-controle $\mathrm{C}$ - livres em gaiolas por 21 dias; $\mathrm{S}$ - suspensos por 21 dias; ST - suspensos pela cauda por 21 dias e simultaneamente tratados com US; $\mathrm{S} \rightarrow \mathrm{C}$ - suspensos por 21 dias, seguidos de 21 dias livres na caixa; $\mathrm{S} \rightarrow \mathrm{CT}-$ suspensos por 21 dias, seguidos de 21 dias livres na caixa com simultâneo tratamento com US. *Letras diferentes significam diferença estatística $(\mathrm{p}<0,05)$.

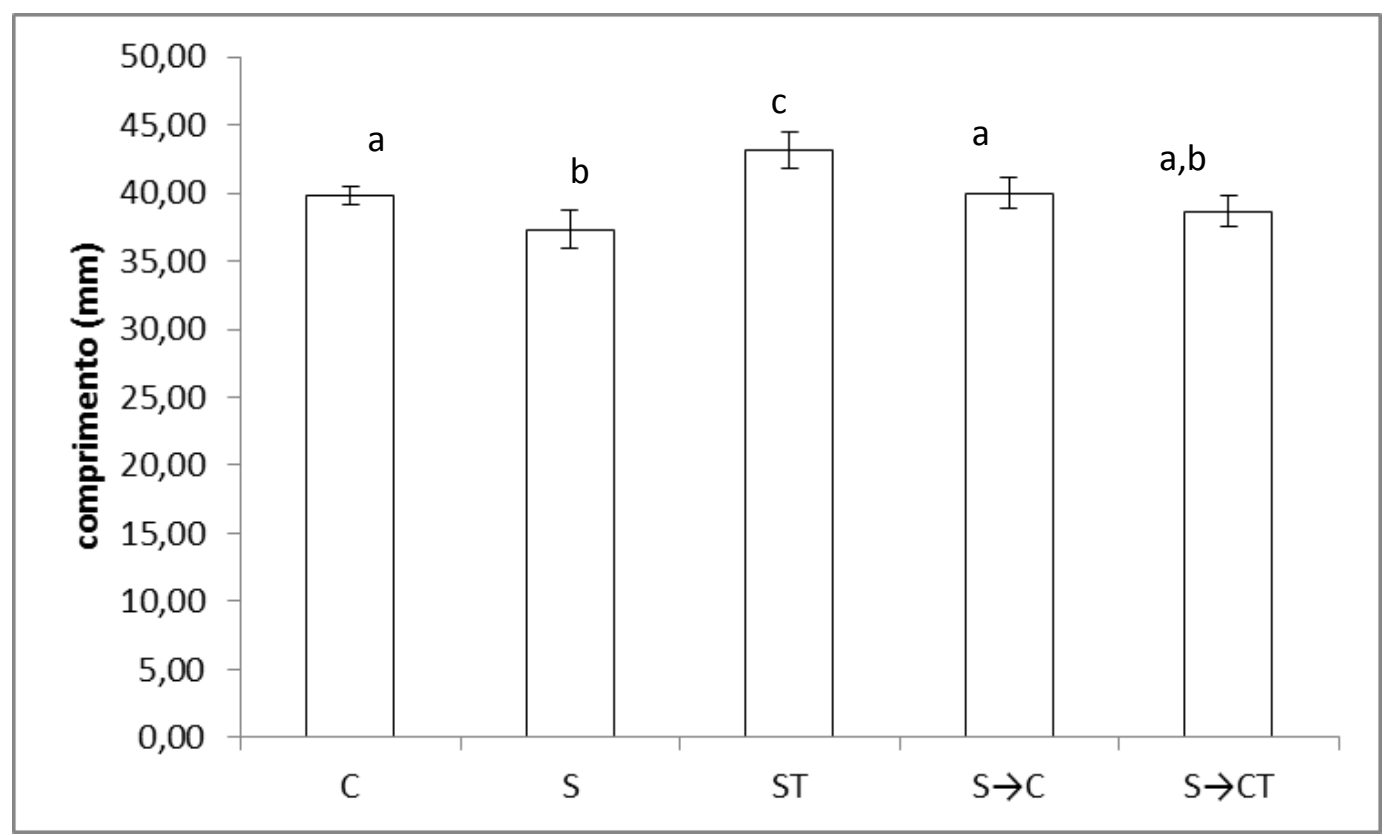

Figura 2. Comprimento das tíbias (média \pm desvio-padrão) de ratos Wistar de animais-controle $\mathrm{C}-$ livres em gaiolas por 21 dias; S - suspensos por 21 dias; ST - suspensos pela cauda por 21 dias e simultaneamente tratados com US; $\mathrm{S} \rightarrow \mathrm{C}$ - suspensos por 21 dias, seguidos de 21 dias livres na caixa; $\mathrm{S} \rightarrow \mathrm{CT}$ - suspensos por 21 dias, seguidos de 21 dias livres na caixa com simultâneo tratamento com US. *Letras diferentes significam diferença estatística $(\mathrm{p}<0,05)$. 


\section{DISCUSSÃO}

Pelas análises estatísticas, ficou demonstrado que o grupo $\mathrm{S}$ apresentou valores para DMO, CMO, Fmáx, rigidez (R), comprimento (L) e diâmetro (D) ósseo menores que os do grupo $\mathrm{C}$, indicando que a suspensão pela cauda é prejudicial à manutenção das propriedades ósseas. Isto está de acordo com a literatura que enfatiza que a ausência de contato com o solo pode ser suficiente para promover alteração óssea (Amadei et al., 2006), tendo como consequência provável a osteoporose (Vargas et al., 2003), doença metabólica em que os ossos se tornam menos resistentes e susceptíveis a fraturas. Nesta condição patológica, a quantidade de tecido ósseo é menor, decorrendo da diminuição na formação óssea, do aumento na reabsorção do osso formado, ou da combinação dos dois (Carvalho et al., 2002).

Os resultados apresentados pelo presente estudo corroboram os de Silva e Volpon (2004), que utilizaram também o método de suspensão pela cauda e concluíram que a hipoatividade reduziu a maioria das propriedades mecânicas dos ossos, enfraquecendo-os.

Ao ser realizado o tratamento dos animais suspensos com o US pulsado de baixa intensidade, foram encontrados para este grupo (ST) valores de CMO e DMO maiores que os do grupo $\mathrm{S}$ e iguais ao do $\mathrm{C}$. Estes resultados foram observados também por Lim et al. (2011), em seus estudos com ratas ovariectomizadas, os quais indicaram que o US pode aumentar a formação e inibir a reabsorção óssea, podendo conter o progresso contínuo da perda de massa óssea e tornar maior o processo de formação, suprindo, assim, o processo de reabsorção e podendo ser eficaz na prevenção da perda de massa óssea.

Ao se comparar a Fmáx, a rigidez $(\mathrm{R})$ e o comprimento (L) dos ossos do grupo ST com o S, foi observado um aumento desses parâmetros, indicando que o US de baixa intensidade foi capaz de aumentar a resistência óssea. Quando comparado o grupo ST com o C, observou-se também um aumento significativo destes valores, e isto está em concordância com os resultados de Woo et al. (2010), em estudos com ratas ovariectomizadas, os quais concluíram que a estimulação com o US pulsado pode ser capaz de diminuir o risco de o osso osteoporótico fraturar, aumentando suas propriedades mecânicas.

Os achados do presente estudo encontram suporte na literatura que relata que o US gera modificações elétricas em nível celular, o que permite maior captação de nutrientes e maior influxo de íons cálcio nas células, aumentando seu potencial eletroquímico (Erdogan e Esen, 2009). Este efeito é chamado efeito piezoelétrico, que induz a reabsorção óssea (Kokubu et al., 1999).

Outros efeitos documentados sobre a aplicação do US de baixa potência na cicatrização óssea incluem aumento expressivo do fator $\beta$ transformador de crescimento, fator importante envolvido na formação do osso quanto à proliferação de osteoblastos e na síntese da matriz óssea, ocorrendo também um aumento do fluxo de íons potássio nos espaços intracelulares (Yonemori et al., 1996; Rubin, 2001; Erdogan e Esen, 2009). Contudo, outros estudos sugerem que a osteogênese pode ser estimulada pelo simples contato do transdutor do US com a região de tratamento (estímulo mecânico), sem influências do estímulo elétrico (Carvalho et al., 2002).

Em discordância dos resultados apresentados pelo presente estudo, Warden et al. (2006), em estudo com fêmures de ratos tratados com US pulsado por 16 e 27 dias, mostraram não haver beneficios na utilização do US no tratamento da osteoporose porque, segundo seus estudos, o osso com osteoporose pode ser menos sensível ao US pelo aumento dos espaços trabeculares.

Outro resultado obtido neste estudo demonstra que a diminuição da massa óssea provocada pela suspensão pela cauda (S) pode ser revertida simplesmente pela ação da descarga do peso corporal $(\mathrm{S} \rightarrow \mathrm{C}$ ) quando os animais estão livres na caixa. Fato a se destacar é que o grupo que recebeu tratamento com US simultaneamente a sua permanência livre na caixa, após período de suspensão $(\mathrm{S} \rightarrow \mathrm{CT})$, não teve diferenças significantes dos parâmetros com o grupo $\mathrm{S} \rightarrow \mathrm{C}$ (que não recebeu tratamento com US).

Os animais suspensos e tratados simultaneamente com o US pulsado (ST), 
entretanto, apresentaram diferenças significantes em relação àqueles somente suspensos pela cauda (S), demonstrando a eficácia do tratamento com o US. Estes resultados corroboram os de Apolinário et al. (2011), que concluíram que a impossibilidade do estímulo mecânico causada pela não deambulação, ou seja, ausência de carga, pode ser minimizada pela ação do US.

Todos os resultados obtidos neste estudo para o $\mathrm{ST}$ e o $\mathrm{S} \rightarrow \mathrm{CT}$ se assemelham também aos de Perry et al. (2009), que, ao analisarem os efeitos do US aplicado em cúbito ulnar do antímero esquerdo em ratas Wistar, demonstraram que os efeitos da estimulação do US pulsado de baixa intensidade imitam as ações de carga mecânica fisiológica quando aplicada ao osso. Corroboram também com Lim et al. (2011), que, em estudo com ratas ovariectomizadas, demonstraram que o US pode conter o progresso contínuo da perda de osso e suprir o processo de formação e reabsorção, sendo eficaz na prevenção da perda óssea e, assim, diminuir o risco de fraturas.

\section{CONCLUSÃO}

Os resultados obtidos neste estudo, com a metodologia proposta, permitem concluir que o ultrassom pulsado de baixa potência contribuiu na prevenção e reversão da ocorrência da osteopenia em animais submetidos à suspensão pela cauda, demonstrando que a ausência do estímulo mecânico causada pela impossibilidade da deambulação pode ser minimizada pela ação mecânica deste.

\section{REFERÊNCIAS}

AMADEI, S.U.; SILVEIRA, V.A.S.; PEREIRA, A.C. et al. A influência da deficiência estrogênica no processo de remodelação e reparação óssea. J. Braz. Patol. Med. Lab., v.42, p.5-12, 2006.

APOLINÁRIO, J.C. Efeito do ultra-som de baixa potência na reparação óssea em ratos sob ausência de carga. Análise densitométrica e biomecânica, 2008. 66f. Dissertação (Mestrado em Ciência Animal) - Faculdade de Medicina Veterinária, Universidade Estadual Paulista/ UNESP - Araçatuba.

APOLINÁRIO, J.C.; COELHO, W.M.D.; LOUZADA, M.J.Q. Análise da influência do ultrassom de baixa intensidade na região de reparo ósseo em ratos sob ausência de carga. Rev. Fisioter. Pesq., v.18, p.275-279, 2011.

ARANHA, L.L.M.; MIRÓN, C.J.A.; ALONSON, S.M. et al. Qualidade de vida relacionada à saúde em espanholas com osteoporose. Rev. Saúde Públ., v.40, p.298-303, 2006.

AZUMA, Y.; ITO, M.; TAKAGI, H. et al. Lowintensity pulsed ultrasound accelerates rat femoral fracture healing by acting on the various cellular reactions in the fracture callus. J. Bone Miner Res., v.16, p.671-680, 2001.

CARVALHO, D.C.L.; ROSIN, C.; GAMA, L.O.R. et al. Tratamentos não farmacológicos na estimulação da osteogênese. Rev. Saúde Pub., v.36, p.647-654, 2002.

DEQUERKER, J.; ORTNER, D.; STIX, A. et al. Hip fracture and osteoporosis in a XIIth Dynasty female skeleton from Lisht, upper Egypt. J. Bone Miner. Res., v.12, p.881-888, 1997.

ENGERMANN, M.; SCHNEIDER, E.; EVANS, C.H.; BALTZER, A.W. The potential of gene therapy for fracture healing in osteoporosis. Osteoporosis Int., v.15, p.82-87, 2005.

ERDOGAN, O.; ESEN, E. Biological aspects and clinical importance of ultrasound therapy in bone healing. J. Ultrasound Med., v.28, p.765776, 2009.

FORSBACH, G.; SANTOS, A. Densidade óssea y osteporosis: una opinion. Ginecol. Obstet., v.62, p.201-203, 1994.

GONÇALVES, R.C.; GONÇALVES, R.L.; BALDUCCI, R.E. Efeitos do ultrassom de baixa intensidade em defeito ósseo do ramo da mandíbula. Estudo histológico em ratos. Rev. Odont. UNESP, v.36, p.193-199, 2007.

JOHNSON, R.B. The bearable lightness of being: bones, muscles, and spaceflight. Anat. Rec., v.253, p.24-27, 1998.

KOKUBU, T.; MATSUI, N.; FUJIOKA, H. et al. Low intensity pulsed ultrasound exposure increases prostaglandin E2 production via the induction of cyclooxy-genase-2 mRNA in mouse osteoblasts. Biochem. Biophys. Res. Commun., v.256, p.284-287, 1999.

LIM, D.; KO, C.Y.; SEO, D.H. et al. Low Intensity Ultrasound Stimulation Prevents Osteoporotic Bone Loss in Young Adult 
Ovariectomized Mice. J. Orthop. Res., v.29, p.116-125, 2011.

LIND, M.; BUNGER, C. Factors stimulating bone formation. Eur. Spine J., v.10, suppl. 2, p.102-109, 2001.

LIRANI, A.P.R. Estudo comparativo dos efeitos do ultra-som e do laser de baixa intensidade no reparo ósseo de tíbia de rato. 2004. 94f. Dissertação (Mestrado em Bioengenharia) Escola de Engenharia de São Carlos, Faculdade de Medicina de Ribeirão Preto e Instituto de Química de São Carlos, Universidade de São Paulo, São Carlos.

MCARDLE, W.D.; KATCH, F.I.; KATCH, V.L. Fisiologia do exercício: energia, nutrição e desempenho humano. Rio de Janeiro: Guanabara-Koogan, 2011. 1132p.

MELLO, W.G.; MORAIS, S.R.L.; DORNELLES, R.C.M. et al. Effects of neonatal castration and androgenization on sexual dimorphism in bone, leptin and corticosterone secretion. Bone, v.50, p.893-900, 2012.

MOREY-HOLTON, E.R.; GLOBUS, R.K. Hindlimb unloading of growing rats: a model for predicting skeletal changes during space flight. Bone, v.22, suppl.5, p.83S-88S, 1998.

MUSACCHIA, X.J.; DEAVERS, D.R.; MEININGER, G.A.; DAVIS, T.P. A model for hypokinesia: effects on muscle atrophy in the rat. J. Appl. Physiol., v.48, p.479-486, 1983.

NAVEGA, M.T.; OISHI, J. Comparação da qualidade de vida relacionada à saúde entre mulheres na pós-menopausa praticantes de atividade física com e sem osteoporose. Rev. Bras. Reumatol., v.47, p.258-264, 2007.
NORDIN, M.; FRANKEL, V.H. Biomecânica básica do sistema musculoesquelético. Rio de Janeiro: Guanabara Koogan, 2003. 401p.

OKUNO, E.; CALDAS, I.L.; CHOW, C. Física para Ciências Biológicas e Biomédicas. São Paulo: Harbra, 1986. 490p.

PERRY, M.J.; PARRY, L.K.; BURTON, V.J. et al. Ultrasound mimics the effect of mechanical loading on bone formation in vivo on rat ulnae. Med. Eng. Phys., v.31, p.42-47, 2009.

RUBIN, C. The use of low-intensity ultrasound to accelerate the healing of fractures. J. Bone Joint Surg. Am., v.83, p.259-270, 2001.

SILVA, A.V.; VOLPON, J.B. Modelo de suspensão pela cauda e seu efeito em algumas propriedades mecânicas do osso do rato. Acta Ortop. Bras., v.12, p.22-31, 2004.

VARGAS, D.M.; RIGOTE, T.; GUTZ, C. et al. Mineralização óssea em crianças e adolescentes com diabetes melito tipo1. J. Pediatr., v.79, p.253-258, 2003.

WARDEN, S.J.; FUCHS, R.K.; KESSLER, C.K. et al. Ultrasound produced by a conventional therapeutic ultrasound unit accelerates fracture repair. Phys Ther., v.86, p.1118-1127, 2006.

WOO, D.G.; KO, C.Y.; KIM, H.S. et al. Evaluation of the Potential Clinical Application of Low-Intensity Ultrasound Stimulation for Preventing Osteoporotic Bone Fracture. Ann. Biomed. Eng., v.38, p.2438-2446, 2010.

YONEMORI, K.; MATSUNAGA, S.; ISHIDOU, Y. et al. Early effects of electrical stimulation on osteogenesis. Bone, v.19, p.173-180, 1996. 